18,12

\title{
Углеродные наноструктуры на полупроводниковой подложке
}

\author{
(C) С.Ю. Давыдов
}

Физико-технический институт им. А.Ф. Иофрфе РАН,

Санкт-Петербург, Россия

E-mail: Sergei_Davydov@mail.ru

Поступила в Редакцию 17 января 2019 г.

В окончательной редакции 17 января 2019 г.

Принята к публикации 31 января 2019 г.

Для простых моделей углеродных наноструктур (латеральной гетероструктуры графен - нитрид бора, декорированных зигзагообразных кромок полубесконечного графена и графеновой наноленты, декорированного карбина) получены аналитические выражения для плотностей состояний и чисел заполнения. Основное внимание уделено режиму сильной связи наноструктур с полупроводниковой подложкой. Численные оценки приведены для $\mathrm{SiC}$-подложки.

DOI: 10.21883/FTT.2019.06.47701.359

\section{1. Введение}

Среди различных изучаемых в настоящее время наноструктур популярны углеродные образования двух типов. Это, в первую очередь, латеральные гетероструктуры (ЛГС), представляющие собой контакт расположенных в одной плоскости листов графена и какого-либо двумерного соединения с гексагональной решеткой, расчетам электронных характеристик которых посвящены работы [1-5]. В этих работах ЛГС рассматривались как свободные, а наличие какой-либо подложки полностью игнорировалось, хотя в реальных структурах без подложки не обойтись. Впервые, насколько известно автору, задача об эпитаксиальных ЛГС на металлической подложке была поставлена в [6,7]. Ко второму типу интенсивно исследуемых в настоящее время углеродных нанообъектов относятся графеновые островки и наноленты, декорированные инородными частицами. Расчеты электронных характеристик таких структур, находящихся в свободном состоянии, выполнены в работах [8-10], роль металлической подложки рассматривалось в [11-13]. Отметим, что работы [1-5,8-10] представляют собой численные расчеты, тогда как в [6,7,11-13] предложены достаточно простые модели (кластерные и цепочечные), позволяющие получить результаты в аналитическом виде. Цепочечные модели будут использованы и в настоящей работе, где в качестве подложки для перечисленных выше наноструктур выбран полупроводник. Мы будем рассматривать зигзагообразные кромки углеродных образований, представляющие, в силу присущей этим кромкам повышенной плотности состояний в окрестности точки Дирака, наибольший интерес. При этом будем применять адсорбционный подход к задаче об эпитаксиальных структурах, выстраивая эти структуры не из свободных атомов с последующим учетом их взаимодействия с подложкой, а из адатомов $[14,15]$.

\section{2. Латеральная гетероструктура графен-графенободобное соединение}

Воспользовавшись решеткой типа кирпичной стенки $[16,17]$, представим зигзагообразный контакт эпитаксиальных графеноподобных соединений $A B$ и $C D$ в виде двух связанных цепочек (рис. 1). Под атомами $A$ и $C$ будем понимать катионы с энергиями $p$-состояний $\varepsilon_{a}$ и $\varepsilon_{c}$, под адатомами $B$ и $\mathrm{D}-$ анионы с энергиями $p$-состояний $\varepsilon_{b}$ и $\varepsilon_{d}$. Будем считать, что не связанным друг другом адатомам $A, B, C$ и $D$ соответствуют

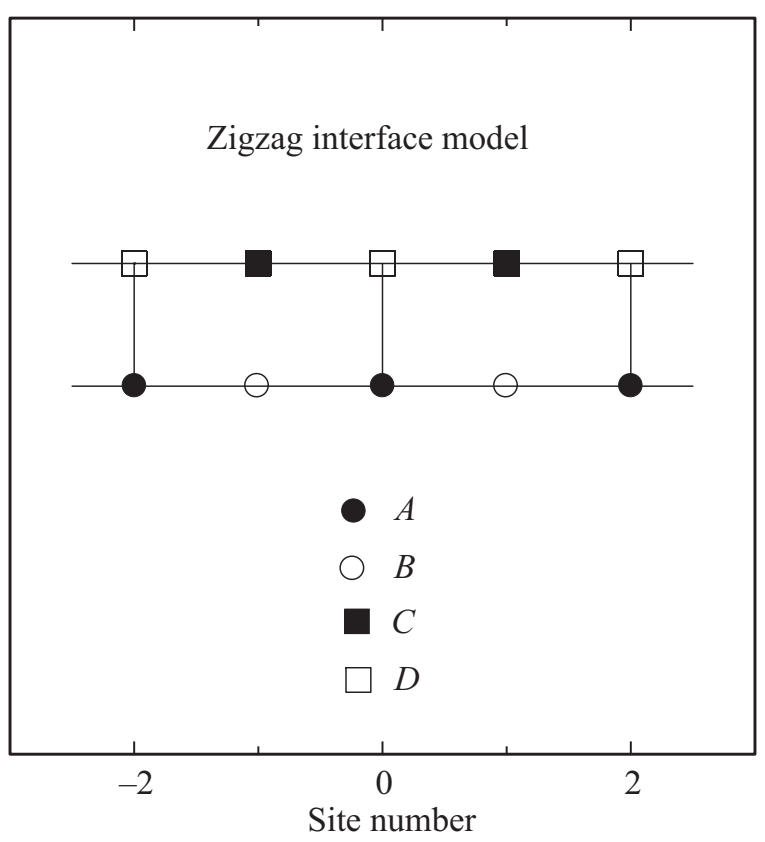

Рис. 1. Структурная модель зигзагообразного контакта графеноподобных структур $A B$ и $C D$. 
затравочные функции Грина:

$$
g_{A, B, C, D}^{-1}(\omega)=\omega-\varepsilon_{a, b, c, d}-\Lambda(\omega)+i \Gamma(\omega) .
$$

Здесь $\Gamma(\omega)=\pi \rho_{s c}(\omega) V^{2}$ и $\Lambda(\omega)=\pi^{-1} P \int_{-\infty}^{\infty} \Gamma\left(\omega^{\prime}\right) d \omega^{\prime} /$ $\left(\omega-\omega^{\prime}+i s\right)-$ функции уширения и сдвига квазиуровня адатома, $\omega-$ энергетическая переменная, $\rho_{s c}(\omega)-$ плотность состояний полупроводниковой подложки, $V-$ матричный элемент взаимодействия адатом-подложка, усредненный по зоне Бриллюэна подложки [14], $P-$ символ главного значения, $s=0^{+}$. Воспользовавшись результатами работы [7], запишем функции Грина для адатомов $A$ и $D(C)$ в виде:

$$
\begin{gathered}
G_{A}(\omega, k)=g_{A}\left(P-g_{A} g_{D} Q^{-1} \tilde{t}^{2}\right)^{-1}, \\
G_{D(C)}(\omega, k)=g_{D}\left(Q-g_{A} g_{D(C)} P^{-1} \tilde{t}^{2}\right)^{-1},
\end{gathered}
$$

где $P=1-g_{A} g_{B} \Phi t^{2}, Q=1-g_{C} g_{D} \Phi t^{2}, \Phi=4 \cos ^{2} k a$, $t$ и $(\tilde{t})$ - энергии перескока электрона между ближайшими соседями (б. с.) внутри цепочек и между цепочками, $k-$ волновой вектор для движения электрона вдоль цепочки $(|k| \leq \pi / 2 a), a-$ расстояние между б.с. в цепочке. Соответствующие функциям Грина (2) плотности состояний и числа заполнения атомов при нулевой температуре равны $\rho_{A, D}(\omega)=-\pi^{-1} \operatorname{Sp} \operatorname{Im} G_{A, D}(\omega, k)$ и $n_{A, D}=2 \int_{-\infty}^{E_{F}} \rho_{A, D}(\omega) d \omega$, где $E_{F}-$ уровень Ферми. В соответствии с [7], будем именовать такую модель ЛГС цепочечной моделью. В дальнейшем в качестве примера рассмотрим контакт графена $(\mathrm{Gr})$ с гексагональным нитридом бора $(h$-BN). Воспользовавшись таблицами атомных термов Манна $[18]$ и отсчитывая энергию от точки Дирака графена $\varepsilon_{D}$ (т.е. принимая $\varepsilon_{D}=\varepsilon_{a}=\varepsilon_{b}=0$ ), получим $\varepsilon_{c} \approx t$ (атом В), $\varepsilon_{d} \approx-t($ атом $\mathrm{N})$, где $t=2.8 \mathrm{eV}$ [19]. Такая оценка согласуется с работами выхода графена и гексагонального нитрида бора, которые практически одинаковы и равны $4.6 \mathrm{eV}[1]$.

Для полупроводниковой подложки выберем модель плотности состояний Халдейна-Андерсона $[14,15]$ : $\rho_{s c}(\omega)=\rho_{s c}=$ const при $|\Omega|>E_{g} / 2$ и 0 при $|\Omega| \leq E_{g} / 2$, где $\Omega=\omega-\varepsilon_{0}, \quad \varepsilon_{0}-$ энергия центра запрещенной зоны, ширина которой равна $E_{g}$. Тогда получим: $\Gamma(\omega)=\pi \rho_{s c} V^{2}=\Gamma$ при $|\Omega|>E_{g} / 2$ и 0 при $|\Omega| \leq E_{g} / 2$, $\Lambda(\omega)=(\Gamma / \pi) \ln \left|\left(\Omega-E_{g} / 2\right) /\left(\Omega+E_{g} / 2\right)\right|$.

Положения центров запрещенных зон $\mathrm{SiC}$-политипов относительно вакуума приведены в таблице (см. [20] и ссылки, приведенные там). Легко видеть, что точка Дирака графена перекрывается с запрещенной зоной карбида кремния. Это наиболее интересная ситуация, так как при попадании точки Дирака в глубину валентной зоны или зоны проводимости мы, по сути, возвращаемся к случаю металлической подложки.
Энергии центров запрещенных зон $\varepsilon_{0}$ относительно вакуума для политипов карбида кремния

\begin{tabular}{c|c|c|c|c|c|c|c}
\hline Политип & $3 C$ & $8 H$ & $21 R$ & $6 H$ & $15 R$ & $27 R$ & $4 H$ \\
\hline $\begin{array}{c}-\varepsilon_{0},{ }^{э} \mathrm{~B} \\
\mathrm{CBOA}\end{array}$ & 5.20 & 5.01 & 5.00 & 4.95 & 4.86 & 4.84 & 4.79 \\
\hline $\begin{array}{c}-\varepsilon_{0},{ }^{\circ B} \\
\text { VBOA }\end{array}$ & 5.20 & 5.00 & 4.96 & 4.94 & 4.92 & 4.90 & 4.86
\end{tabular}

Приме чание. Использованы приближения CBOA (conduction band offset approximation) и VBOA (valance band offset approximation).

Рассмотрим режим слабой связи ЛГС с подложкой $(\Gamma / t \ll 1)$. Как и в $[6,7]$, можно показать, что в приближении бездисперсионных зон $(\Phi(k)=\bar{\Phi}=2)$ плотности состояний $\rho_{A, D}(\omega)$ и числа заполнения $n_{A, D}=2 \int_{-\infty}^{E_{F}} \rho_{A, D}(\omega) d \omega$ могут быть представлены в виде

$$
\rho_{A, D}(\omega)=\sum_{j}\left(v_{A, D}\right)_{j} \rho_{j}(\omega), \quad n_{A, D}=2 \sum_{j}\left(v_{A, D}\right)_{j} n_{j} .
$$

Здесь $\left(v_{A, D}\right)_{j}-$ весовые коэффициенты, равные вычетам функций Грина (2) в полюсах $E_{j}$, где индекс $j=1,2,3,4$ нумерует зоны (см. подробнее $[6,7]$ ); для области энергий, отвечающих сплошному спектру, зонные вклады в плотности состояний и числа заполнения равны $\rho_{j}(\omega)=\Gamma / \pi\left[\left(\omega-\Lambda(\omega)-E_{j}\right)^{2}+\Gamma^{2}\right]$ и $n_{j}=$ $=\pi^{-1} \operatorname{arccot}\left[\left(E_{j}+\Lambda\left(E_{j}\right)+E_{g / 2}\right) / \Gamma\right]$; для области запрещенных состояний соответствующие локальные вклады есть $\rho_{j}=\delta\left(\omega-E_{j l}-\Lambda\left(E_{j l}\right)\right)$ и $n_{j}=\Theta\left(E_{F}-E_{j l}-\Lambda\left(E_{j l}\right)\right)$, где $E_{j l}-$ энергия локального уровня, а функции $\delta(\ldots)$ и $\Theta(\ldots)$ - дельта-функция Дирака и функция Хэвисайда.

Плотности состояний на адатомах углерода, азота и бора для эпитаксиальной ЛГС $(\mathrm{Gr}-h$-BN $) @ 4 H-\mathrm{SiC}$ в режиме слабой связи представлены на рис. 2, $a$ для области энергий, отвечающих валентной зоне подложки. Обращают на себя внимание три пика. Два из них отвечают энергиям $\sim E_{j}$, отражая, таким образом, зонную структуру свободной ЛГС $\mathrm{Gr}-h$-BN [7]. Третий пик, появляющийся вследствие связи с полупроводниковой подложкой, лежит вблизи потолка валентной зоны при энергии $-E_{g} / 2-\xi_{j}$, где $\xi_{j} \sim E_{g} \exp \left(-\pi \Delta_{j} / \Gamma\right)$, $\Delta_{j}=\left|E_{j}\right|-E_{g} / 2$, откуда следует, что $\xi_{j} \ll E_{g} / 2$.

$\mathrm{B}$ [7] для свободной структуры $\mathrm{Gr}-h$ - $\mathrm{BN}$ в приближении бездисперсионных зон при $\tau=\widetilde{(t}) / t=1$ было получено: $n_{A}^{0}=n_{B}^{0}=n_{\mathrm{Gr}}^{0}=1, \quad n_{D}^{0}=n_{\mathrm{N}}^{0}=1.55$. $n_{C}^{0}=n_{\mathrm{B}}^{0}=0.45$. При $\varepsilon_{D}=\varepsilon_{0}=E_{F}=0$, для адсорбционной системы $(\mathrm{Gr}-h$ - $\mathrm{BN}) @ 4 H-\mathrm{SiC}$ получим $n_{\mathrm{CV}}=0.97$ (для адатомов $A$ и $B$ ), $n_{\mathrm{N} V}=1.49, n_{\mathrm{B} V}=0.44$. Энергии локальных уровней $E_{j l}$ для $j=3,4$ в режиме слабой связи двумерных слоев с подложкой равны $E_{j l} \approx-E_{g} / 2+\xi_{j}$, где $\xi_{j} \approx E_{g} \exp \left(-\pi \Delta_{j} / \Gamma\right)$, $\Delta_{j}=\left|E_{j}\right|-E_{g} / 2$. При этом значения локальных вкладов $n_{j l} \approx\left(1+\Gamma / 2 \pi \xi_{j}\right)^{-1}$ исчезающе малы [14,15]. Таким 

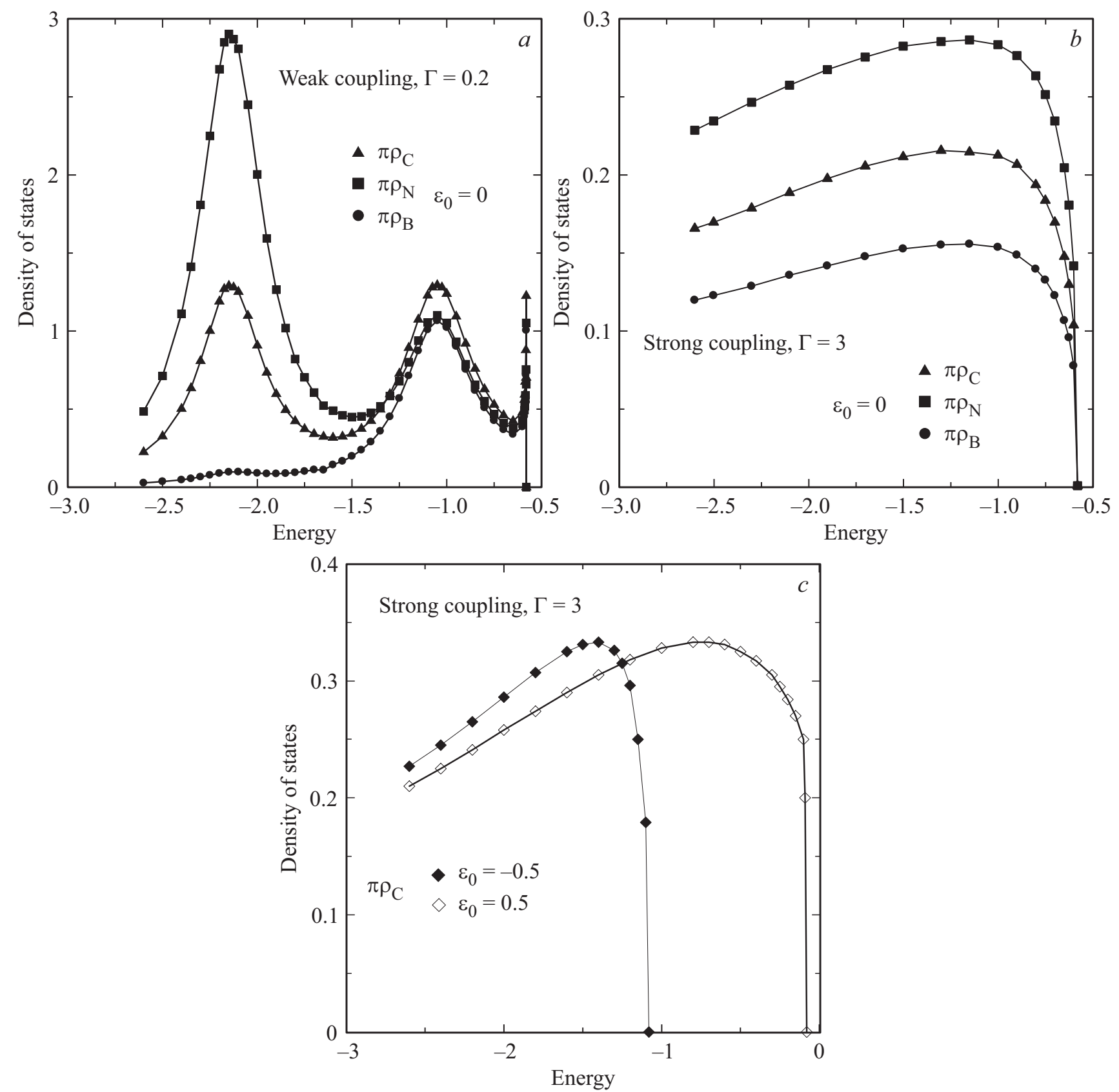

Рис. 2. Латеральная гетероструктура $(\mathrm{Gr}-h$-BN)@4H-SiC: плотности состояний на адатомах $\mathrm{C}, \mathrm{N}$ и В для режимов слабой $(a)$ $(\Gamma=0.2 t)$ и сильной $(b, c) \quad(\Gamma=3 t)$ связи гетероструктуры $\mathrm{Gr}-h$-BN с $\operatorname{SiC}$-подложкой при $t=\tilde{t}, \varepsilon_{D}=\varepsilon_{0}=0 \quad(a, b)$ и $t=\tilde{t}$, $\varepsilon_{D}=0, \varepsilon_{0}= \pm 0.5 t(c)$. Все энергетические характеристика измерены в ед. $t$, плотности состояний - в ед. $t^{-1}$. На рисунке представлены только левые части зависимостей $\rho_{\mathrm{C}, \mathrm{B}, \mathrm{N}}(\omega)$, так как $\rho_{\mathrm{C}}(\omega)=\rho_{\mathrm{C}}(-\omega), \rho_{\mathrm{B}, \mathrm{N}}(\omega, t)=\rho_{\mathrm{N}, \mathrm{B}}(-\omega,-t)$ при $\varepsilon_{D}=\varepsilon_{0}=0$ и $\rho_{\mathrm{C}}\left(\omega, \varepsilon_{0}\right)=\rho_{\mathrm{C}}\left(-\omega,-\varepsilon_{0}\right)$ при $\varepsilon_{D}=0, \varepsilon_{0} \neq 0$. Состояния в запрещенной зоне на рисунке не изображены.

образом, в пределе слабой связи в адсорбционной системе $(\mathrm{Gr}-h$-BN $) @ 4 H-\mathrm{SiC}$ суммарные числа заполнения определяются валентной зоной. При этом имеет место переход малого числа электронов (0.01 e/atom) с ЛГС в подложку.

Так как по нашим оценкам имеем $\varepsilon_{0} / t \sim 0.1$ (см. таблицу), положим $\left|\varepsilon_{0}\right| \ll\left|E_{j}\right|$. Легко показать, что $\Lambda\left(E_{j}, \varepsilon_{0}\right) \approx \Lambda\left(E_{j}, 0\right)-\lambda$, где при принятых нами параметрах $\lambda=\varepsilon_{0} E_{g} \Gamma / \pi\left(E_{j}^{2}-E_{g}^{2} / 4\right) \ll \Gamma$. Отсюда следует, что поправка порядка $\lambda \Gamma /\left(E_{j}+\Lambda\left(E_{j}\right)+E_{g} / 2\right)^{2}$ к вкладу валентной зоны в числа заполнения пренебрежимо мала. То же имеет место и для локальных вкладов.

Отметим, что полученные здесь для полупроводниковой подложки числа заполнения весьма близки к числам заполнения ЛГС $\mathrm{Gr}-h$-BN как в свободном состоянии, так и при наличии металлической подложки (см. [7], рис. 3). Такой результат можно объяснить следуюшим образом. Во-первых, функция сдвига $\Lambda(\omega)$ 
для полупроводника в режиме слабой связи достигает больших значений только в очень узких областях вблизи потолка валентной зоны $E_{V}$ и дна зоны проводимости $E_{C}[14,15,20,21]$ (в модели параболической плотности состояний, в отличие от модели Халдейна-Андерсона, такие области вообще отсутствуют). Вне этих областей можно считать $\Lambda(\omega) \propto \Gamma \sim 0$, что сводит задачу о ЛГС на полупроводнике к случаям ЛГС в свободном состоянии и на металлической подложке, где функции $\Lambda(\omega)$ тождественно равны нулю $[14,15]$. Во-вторых, энергии локальных уровней равны $E_{j l} \approx \mp E_{g} / 2 \pm \xi_{j l}$, где $\xi_{j} \approx E_{g} \exp \left(-\pi \Delta_{j} / \Gamma\right)$ и $\Delta_{j} \approx\left|E_{j}\right|-E_{g} / 2$, так что локальные вклады $n_{j l} \approx\left(1+\Gamma / 2 \pi \xi_{j}\right)^{-1}$ в суммарные числа заполнения исчезающе малы. Именно поэтому в режиме слабой связи полагают зачастую, что плотность состояний эпиграфена та же что и у свободного графена, а его заполнение диктуется исключительно химическим потенциалом (уровнем Ферми). Принимая в дальнейшем такую оценку, мы будем особое внимание уделять режиму сильной связи ЛГС с подложкой.

Формулы для плотностей состояний в пределе $t / \Gamma \ll 1, \tilde{t} / \Gamma \ll 1$ приведенные в Приложении, п. 1 , достаточно громоздки. Оценки, однако, показывают, что при $\Gamma \geq 3 t$ с хорошей точностью можно положить

$$
\pi \rho_{A}(\omega) \approx A(\omega), \quad \pi \rho_{C, D}(\omega) \approx A_{ \pm}(\omega)
$$

что соответствуют плотностям состояний изолированных (одиночным) адатомов углерода, азота и бора. Зависимости $\rho_{A, B, C}(\omega)$ представлены на рис. $2, b, c$, откуда следует, во-первых, что проявления зонной структуры ЛГC $\mathrm{Gr}-h$-NB в виде двух пиков при энергиях $\sim E_{j}$ в пределе сильной связи исчезают. Остается только максимум вблизи потолка валентной зоны, присущий как слабой, так и сильной связи. Во-вторых, рис. 2, с демонстрирует, что сдвиг запрещенной зоны подложки относительно точки Дирака $\varepsilon_{D}$ приводит к соответствующему смещению плотности состояний как целого.

Так как при $\Gamma \geq 3 t$ адатомы C, В и $\mathrm{N}$ можно считать изолированными, для оценки вкладов валентной зоны $n_{\mathrm{CV}}, n_{\mathrm{B} V}$ и $n_{\mathrm{N} V}$ в суммарные числа заполнения можно воспользоваться результатом работы [22] и положить $\left.n_{\mathrm{CV}}=(2 / \pi) \operatorname{arccot}(R / \Gamma), \quad n_{\mathrm{N}, \mathrm{B} V}=(2 / \pi) \operatorname{arccot}(R \mp t) / \Gamma\right)$, $R=\sqrt{\left(\Gamma E_{g} / \pi\right)+\left(E_{g}^{2} / 4\right)}$, где, как и выше, $\varepsilon_{D}=\varepsilon_{0}=\varepsilon_{F}=0$. Считая $\Gamma$ самым большим параметром задачи, получим $n_{\mathrm{CV}} \approx 1-(2 / \pi) \sqrt{E_{g} / \pi \Gamma}$ и $n_{\mathrm{N}, \mathrm{B} V} \approx 1-(2 / \pi)$ $\times \sqrt{E_{g} / \pi \Gamma} \mp t / \Gamma$. Для системы $(\mathrm{Gr}-h-\mathrm{BN}) @ 4 H-\mathrm{SiC}$ при $\Gamma=3 t$ имеем $n_{\mathrm{CV}}=0.78, n_{\mathrm{BV}}=0.57$ и $n_{\mathrm{NV}}=0.99$. Решая уравнение $\omega-\Lambda(\omega)=0$ для адатома $C$ и уравнения $\omega \mp t-\Lambda(\omega)=0$ для адатомов $\mathrm{N}$ и $\mathrm{B}$, для $\mathrm{C}$ и $\mathrm{B}$ найдем локальные уровни $\omega_{\mathrm{Cl}}=0$ и $\omega_{\mathrm{B} l}=-0.224 t$, для адатома $\mathrm{N}$ локальное состояние отсутствует. Тогда получаем $n_{\mathrm{Cl}}=0.23$ и $n_{\mathrm{B} l}=0.21$. Окончательно получаем $n_{\mathrm{C}}=1.01$ (как для адатомов типа $A$, так и для адатомов типа $B$, рис. 1$), n_{\mathrm{B}}=0.78$ и $n_{\mathrm{N}}=0.99$. Таким образом, в режиме сильной связи имеет место переход малого числа электронов (0.22 e/atom) с ЛГС в подложку. Следует

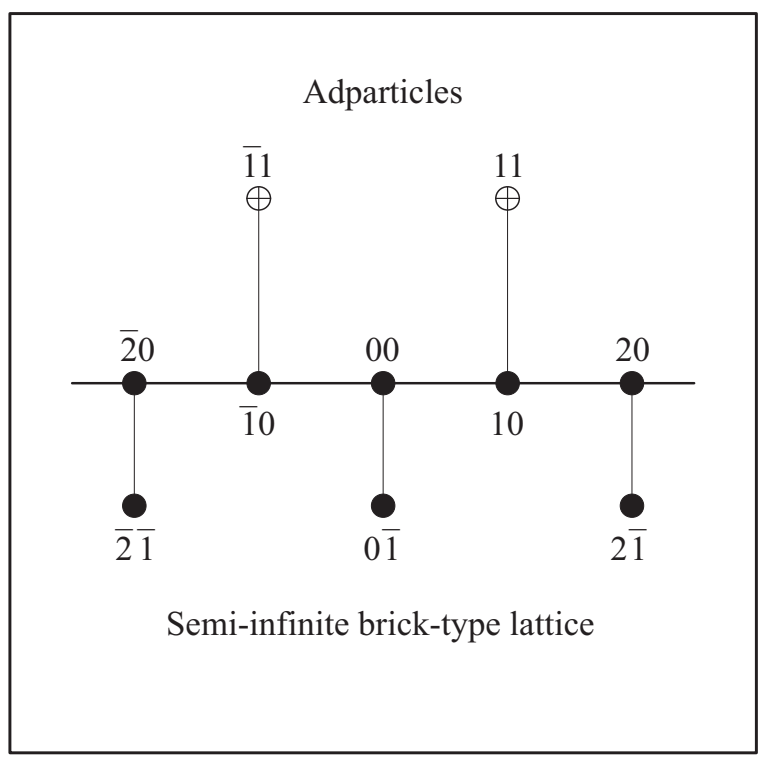

Рис. 3. Структурная модель декорированной зигзагообразной кромки полубесконечного листа графена.

подчеркнуть, что заполнение адатомов углерода $n_{\mathrm{C}} \sim 1$ практически не зависит от силы связи с подложкой, роль которой сводится, в основном, к перераспределению электронов между адатомами бора и азота.

\section{3. Декорированные зигзагообразных кромок углеродных наноструктур}

На рис. 3 изображена структурная модель декорирования кромки полубесконечного листа эпиграфена, для построения которой мы вновь воспользовались решеткой типа кирпичной стенки $[16,17]$ с расстояние $a$ между б.с. Изолированным адатомам углерода и декорирующим адчастицам припишем соответственно затравочные функции Грина

$$
\begin{gathered}
g(\omega)=(\omega-\Lambda(\omega)+i \Gamma(\omega))^{-1}, \\
g_{a}(\omega)=\left(\omega-\varepsilon_{a}-\Lambda_{a}(\omega)+i \Gamma_{a}(\omega)\right)^{-1},
\end{gathered}
$$

где энергия $p$-состояния адатома углерода принята за нуль, $\varepsilon_{a}$ - энергия уровня адчастицы, $\Gamma(\omega)$ и $\Gamma_{a}(\omega)-$ функции уширения квазиуровней адатомов углерода и адчастиц, $\Lambda(\omega)$ и $\Lambda_{a}(\omega)$ - соответствующие функции сдвига квазиуровней. Воспользовавшись для нахождения функций Грина $G$ уравнением Дайсона $G=g+g T G$, где $T$ - оператор взаимодействия [14,15], и нумерацией адатомов $(x, y)$, представленной на рис. 3, получим следующие выражения [12]:

$$
\begin{aligned}
& G_{00,00} \equiv G_{1}(\omega, k)=g\left(1-g^{2} t^{2}-\frac{g^{2} \Phi t^{2}}{1-g g_{a} \tilde{t}^{2}}\right)^{-1}, \\
& G_{10,10} \equiv G_{2}(\omega, k)=g\left(1-g g_{a} t^{2}-\frac{g^{2} \Phi t^{2}}{1-g^{2} t^{2}}\right)^{-1},
\end{aligned}
$$




$$
\begin{gathered}
G_{11,11} \equiv G_{a}(\omega, k)=g_{a}\left(1-\frac{g g_{a} \tilde{t}^{2}}{1-\frac{g^{2} t^{2} \Phi}{1-g^{2} t^{2}}}\right)^{-1}, \\
G_{0-1,0-1} \equiv G_{-1}(\omega, k)=g\left(1-\frac{g^{2} t^{2}}{1-\frac{g^{2} \Phi t^{2}}{1-g g_{a} \tilde{t}^{2}}}\right)^{-1},
\end{gathered}
$$

где энергия перехода между б.с. в графене есть $t$, а между адатомами графена и адчастицами равна $\tilde{t}$. Согласно оценкам работы [12], в свободном состоянии при $\varepsilon_{a}=0.5 t, \tau=\tilde{t} / t=0.5, \Gamma=\Gamma_{a}=0.2 t$ числа заполнения равны $n_{1}^{0}=n_{-1}^{0}=0.79, n_{2}^{0}=1.74, n_{a}^{0}=0.68$. В режиме слабой связи графена и декорирующих частиц с металлической подложкой при $E_{F}=0$ в [12] было получено: $n_{1}=n_{-1}=0.81, n_{1}=n_{-1}=1.40, n_{a}=0.66$. Отсюда следует, что отток электронов в подложку, равный 0.32 e/atom, идет, главным образом, с адатомов углерода типа $(-20),(00)$ и (02) (рис. 3). Как обсуждалось в предыдущем разделе, эти результаты могут служить оценками и для случая слабой связи декорированного графена с полупроводниковой подложкой.

В режиме сильной связи из выражений (6)-(9) с точностью до $t^{2} / \Gamma^{2}$ и $\tilde{t}^{2} / \Gamma^{2}$ получим приближенные значения функций Грина $G_{1}(\omega) \approx g\left(1+3 g^{2} t^{2}\right)$, $G_{-1}(\omega) \approx g\left(1+g^{2} t^{2}\right), G_{a}(\omega) \approx g_{a}\left(1+g g_{a} \tilde{t}^{2}\right), G_{2}(\omega) \approx$ $\approx g_{a}\left(1+2 g^{2} t^{2}+g g_{a} \tilde{t}^{2}\right)$. В дальнейшем полагаем для простоты $\Gamma(\omega)=\Gamma_{a}(\omega)$ и $\Lambda(\omega)=\Lambda_{a}(\omega)$. Аналитические выражения для плотностей состояний $\rho_{n}(\omega)$, где $n=1,2,-1, a$ приведены в Приложении, п. 2. Графики зависимостей $\rho_{n}(\omega)$ для случая $4 H-\mathrm{SiC}$-подложки при $\varepsilon_{D}=\varepsilon_{a}=0, \quad \varepsilon_{a}=0.5 t, \quad \tau=0.5, \quad \Gamma=3 t$ представлены на рис. 4. Отметим, что особенностью функций $\rho_{n}(\omega)$ является их некоторая изломанность по сравнению с гладкими функциями $\rho_{A, B, C}(\omega)$, приведенными на pис. 2, $b, c$. Это различие возникает, с одной стороны, из-за учета поправок к 1 в круглых скобках в формулах (П8)-(П11) при расчете $\rho_{n}(\omega)$ и, с другой стороны, из-за игнорирования поправок в квадратных скобках в выражениях (П1) и (П2) при расчете $\rho_{A, B, C}(\omega)$. Природа подобной изломанности связана с расходимостью функции сдвига $\Lambda(\omega)$ вследствие скачков плотности состояний в модели Халдейна-Андерсона $[14,15]$. Отметим, что значения всех добавок в круглых скобках формул (П8)-(П11) в рассмотренном энергетическом интервале положительны.

Игнорируя такие поправки, положим $\rho_{1}(\omega) \approx \rho_{2}(\omega) \approx$ $\approx \rho_{-}(\omega) \approx \bar{\rho}_{g}(\omega)$ и $\rho_{a}(\omega) \approx \vec{\rho}_{a}(\omega)$. Воспользовавшись популярным в теории адсорбции приемом, когда функцию $\Lambda(\omega)$ заменяют константой $\Lambda\left(\varepsilon_{0}\right)$ [13], при $\varepsilon_{F}=0$ получим $n_{g} \approx 1$ и $n_{a V} \approx(2 / \pi) \operatorname{arccot}\left[\left(\varepsilon_{a}+\Lambda\left(\varepsilon_{a}\right)\right) / \Gamma\right]$. Полагая $\left|\varepsilon_{a}+\Lambda\left(\varepsilon_{a}\right)\right| \ll \Gamma, \quad$ имеем $n_{a V} \approx 1-2$ $\times\left[\left(\varepsilon_{a}+\Lambda\left(\varepsilon_{a}\right)\right) / \pi \Gamma\right]$, так что $n_{a V}>1$ при $\varepsilon_{a}<0$ и $n_{a V}<1$ при $\varepsilon_{a}>0$. Для $\varepsilon_{a}=0.5 t$ и $\Gamma=3 t$ получаем $n_{a V} \approx 0.81$.

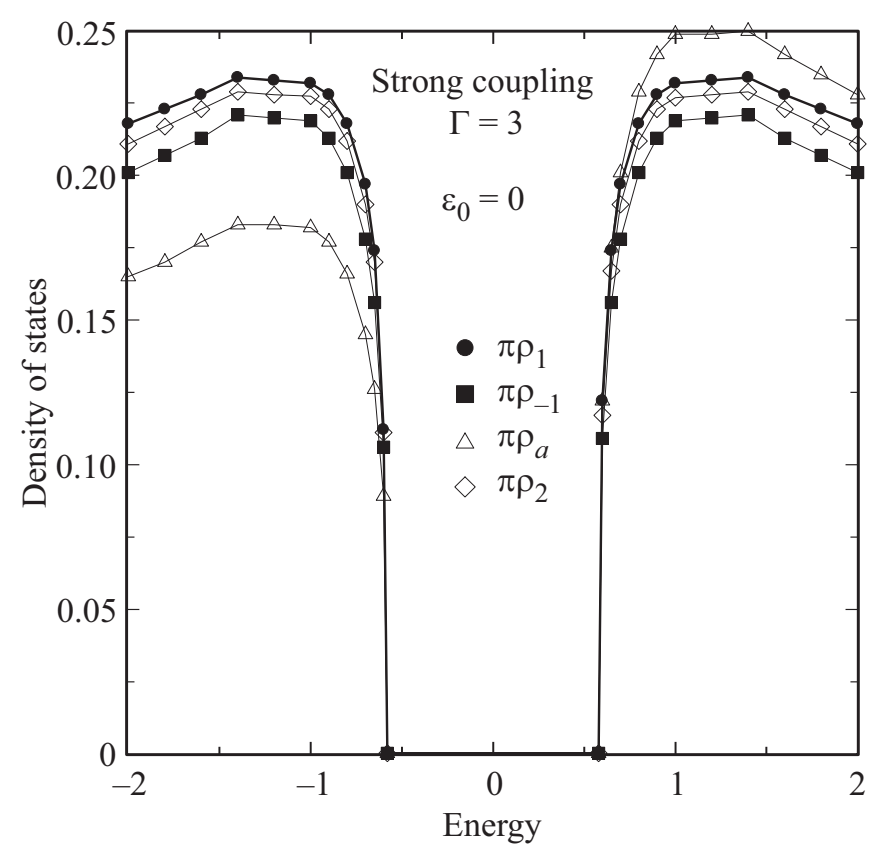

Рис. 4. Декорированная кромка графена: плотности состояний в режиме сильной связи с $4 H$-SiC-подложкой при $\varepsilon_{D}=\varepsilon_{0}=0$, $\varepsilon_{a}=0.5 t, \tau=0.5, \Gamma=3 t$. Все энергетические характеристика измерены в ед. $t$, плотности состояний - в ед. $t^{-1}$. Состояния в запрещенной зоне на рисунке не изображены.

Перейдем теперь к оценкам вкладов локальных состояний в числа заполнения. Для адатомов с $n=1,2,-1$ локальный уровень $\omega_{g l}=0$, так что $n_{g l} \approx 0.23$. Для декорирующей адчастицы при $\varepsilon_{a}=0.5 t$ и $\Gamma=3 t$ получим $\omega_{a l}=0.115 t$ и $n_{g l}=0$, то есть локальный уровень пуст. Так как $\rho_{a}\left(\omega, \varepsilon_{a}\right)=\rho_{a}\left(-\omega,-\varepsilon_{a}\right)$, то положив $\varepsilon_{a}=-0.5 t$, получим $\omega_{a l}=-0.115 t$ и $n_{a l}=0.23$. Таким образом, суммарные числа заполнения равны $n_{g}=1$ и $n_{a}=1.04$. Сравнение с режимом слабой связи показывает, что сильная связь увеличивает заполнение всех адатомов, нивелируя при этом различия для адатомов с $n=1,2,-1$. При заряд $0.04 \mathrm{e} / \mathrm{atom}$ переходит с подложки на наноструктуру.

Обратимся теперь к декорированной наноленте графена с зигзагообразными кромками, структурная модель которой представленная на рис. 5 (см. [13]). Изолированным адатому углерода и адчастице вновь припишем затравочные функции Грина (5). Функции Грина ЛГС имеют вид [13]:

$$
\begin{aligned}
G_{01,01}(\omega, k) & \equiv G_{0}^{\prime}(\omega, k) \\
& =g\left(1-\frac{g^{2} t^{2} \Phi}{1-g g_{a} \tilde{t}^{2}}-\frac{g^{2} t^{2}}{1-\frac{g^{2} t^{2} \Phi}{1-g g_{a} \tilde{t}^{2}}}\right)^{-1},
\end{aligned}
$$




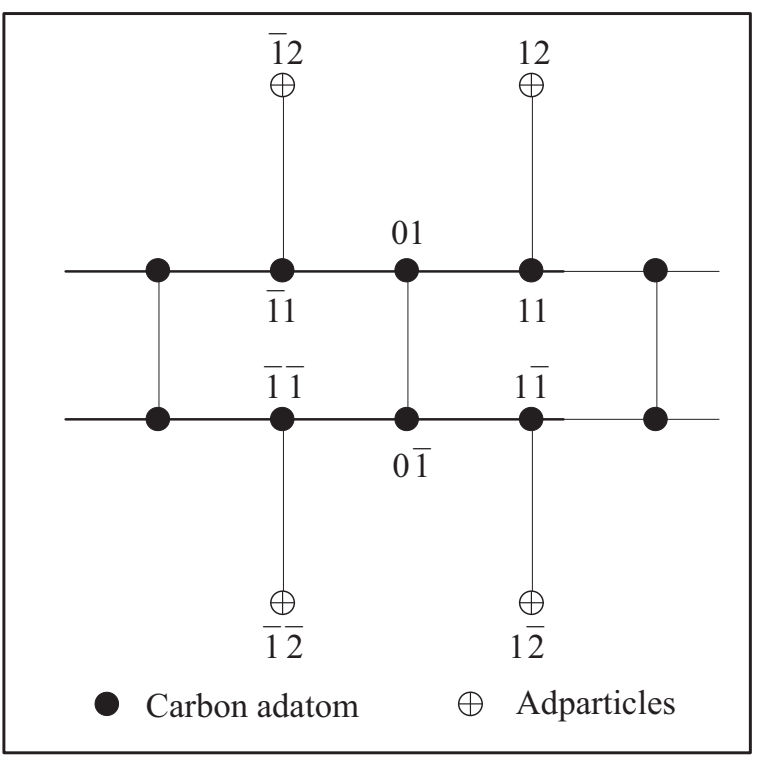

Рис. 5. Структурная модель декорированной наноленты и нумерация адатомов графена и декорирующих адчастиц.

$$
\begin{aligned}
G_{11,11}(\omega, k) & \equiv G_{1}^{\prime}(\omega, k) \\
& =g\left(1-g g_{a} \tilde{t}^{2}-\frac{g^{2} t^{2}}{1-\frac{g^{2} t^{2} \Phi}{1-\frac{g^{2} t^{2} \Phi}{1-g g_{a} \tilde{t}^{2}}}}\right)^{-1},
\end{aligned}
$$$$
G_{12,12}(\omega, k) \equiv G_{a}^{\prime}(\omega, k)
$$

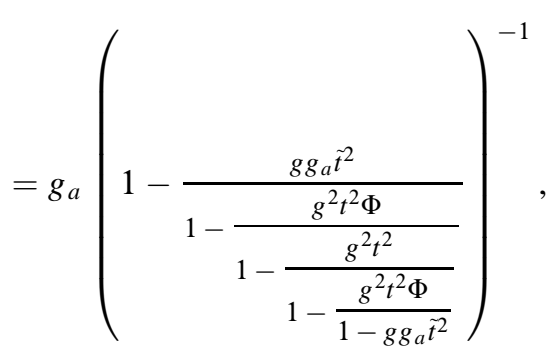

Как показано в [13], в отсутствии подложки числа заполнения всех атомов равны 1. Поэтому основное внимание в [13] уделено режиму сильной связи с металлической подложкой. Для полупроводниковой подложки, сохраняя в (10)-(12) только члены порядка $t^{2} / \Gamma^{2} \quad$ и $\tilde{t}^{2} / \Gamma^{2}$, получим $G_{0}^{\prime}(\omega, k) \approx g\left(1+3 g^{2} t^{2}\right)$, $G_{1}^{\prime}(\omega) \approx g\left(1+2 g^{2} t^{2}+g g_{a} \tilde{t}^{2}\right), \quad G_{a}^{\prime}(\omega) \approx g_{a}\left(1+g g_{a} \tilde{t}^{2}\right)$, Нетрудно видеть, что $G_{0}^{\prime}(\omega)=G_{1}(\omega), G_{1}^{\prime}(\omega)=G_{2}(\omega)$, $G_{a}^{\prime}(\omega)=G_{a}(\omega)$. Таким образом, задача о декорировании кромок наноленты сводится к задаче о декорировании кромки графена в режиме сильной связи с полупроводниковой подложкой.

И, наконец, рассмотрим декорированный карбин (цепочку углеродных адатомов). Соответствующая структурная модель может быть получена путем замены на рис. 3 адатомов графена типа $-2-1,0-1,2-1$ декорирующими адчастицами. Тогда из выражений (6)-(9) получим функции Грина

$$
\begin{aligned}
G^{\prime \prime}(\omega, k) & \equiv G_{0}^{\prime \prime}(\omega, k)=G_{1}^{\prime \prime}(\omega, k) \\
& =g\left(1-g g_{a} \tilde{t}^{2}-\frac{g^{2} t^{2} \Phi(k)}{1-g g_{a} \tilde{t}^{2}}\right)^{-1}, \\
G_{a}^{\prime \prime}(\omega, k) & =g_{a}\left(1-\frac{g g_{a} \tilde{t}^{2}}{1-\frac{g^{2} t^{2} \Phi(k)}{1-g g_{a} \tilde{t}^{2}}}\right)^{-1},
\end{aligned}
$$

которые в режиме сильной связи переходят в уже известные выражения, а именно: $G_{1}^{\prime \prime}(\omega)=G_{1}^{\prime}(\omega)$ и $G_{a}^{\prime \prime}(\omega)=G_{a}^{\prime}(\omega)$

\section{4. Заключительные замечания}

В настоящей работе для цепочечных моделей углеродных эпитаксиальных наноструктур мы обобщили расчетные схемы, предложенные в работах $[7,12,13]$ для металлических подложек, на полупроводниковые подложки. При этом основное внимание было уделено режиму сильной, т. е. ковалентной, связи наноструктуры c подложкой, тогда как под слабой связью следует понимать ван-дер-ваальсово взаимодействие. Для формирования приборных планарных структур предпочтительной (с чисто механической точки зрения) представляется сильная связь, обеспечивающая хорошую адгезию нанослоя. При этом, однако, электронная структура нанообъекта испытывает радикальную перестройку, так что ее особенности практически исчезают. С другой стороны, при сильной связи подложка наводит щель в плотности состояний графена, что является весьма желательной характеристикой реальной приборной структуры (при этом говорят о функционализации графена). Ван-дер-ваальсова связь радикальных изменений электронных характеристик не вызывает, но представляется проблематичной при создании планарных структур.

Мы показали, что для грубых и простых оценок перехода заряда в углеродных наноструктурах можно использовать следующий сценарий: 1) при слабой связи эпиструктуры с подложкой можно полагать числа заполнения адатомов равными числам заполнения соответствующих атомов; 2) при сильной связи адатомы можно считать изолированными и приписывать их числа заполнения числам заполнения адатомов эпиструктуры.

Оценки чисел заполнения показали, что переход заряда может идти в обе стороны: с ЛГС в подложку и с подложки в ЛГС. Возможность как $n$-, так и $p$-типа проводимости эпиграфена на $\mathrm{SiC}$-подложке доказана экспериментально [23]. По-видимому, можно твердо утверждать, что переход заряда мал. Отметим, однако, что переход заряда $\sim 0.01 e$ на атом отвечает величине 
поверхностной плотности заряда $\sigma \sim 6 \cdot 10^{-2} \mathrm{C} / \mathrm{m}^{2}$, которая превышает, например, величину спонтанной поляризации некубических политипов карбида кремния [24].

Отметим в заключение, что модельный подход к задаче об углеродных наноструктурах на металлах и полупроводниках можно сформулировать более строго, как, например, это сделано для графеноподобных соединений $[25,26]$. При этом в случае полупрводниковой подложки в зависимости от выбора параметров модели могут реализовываться весьма различные варианты гетеропереходов между поверхностной наноструктурой и подложкой. Из-за отсутствия необходимой экспериментальной информации, однако, подобное рассмотрение, на наш взгляд, преждевременно.

\section{ПРИЛОЖЕНИЕ}

1. В режиме сильной связи ЛГС с подложкой $(t / \Gamma \ll 1)$ из выражений $(1)$ и $(2)$ получим:

$$
\begin{aligned}
\pi \rho_{A}(\omega) \approx & A(\omega)\left[1+F_{A 1}(\omega)+H_{A 1}(\omega)\right] \\
& +B(\omega)\left[F_{A 2}(\omega)+H_{A 2}(\omega)\right], \\
\pi \rho_{C, D}(\omega) \approx & A_{\mp}(\omega)\left[1+F_{C, D 1}(\omega)+H_{A 1}(\omega)\right] \\
& +B_{ \pm}(\omega)\left[F_{C, D 2}(\omega)+H_{A 2}(\omega)\right],
\end{aligned}
$$

где верхний знак относится к адатому азота, нижний к адатому бора. Выражения для входящих в (П1) и (П2) величин имеют вид

$$
\begin{gathered}
A=\frac{\Gamma}{(\omega-\Lambda)^{2}+\Gamma^{2}}, \quad B=\frac{\omega-\Lambda}{(\omega-\Lambda)^{2}+\Gamma^{2}}, \\
A_{ \pm}=\frac{\Gamma}{(\omega-\Lambda \pm t)^{2}+\Gamma^{2}}, \quad B_{p m}=\frac{\omega-\Lambda \pm t}{(\omega-\Lambda \pm t)^{2}+\Gamma^{2}} \\
F_{A 1}=2 t^{2}\left[(\omega-\Lambda)^{2}-\Gamma^{2}\right] f_{A}, \quad F_{A 2}=4 t^{2} \Gamma(\omega-\Lambda)^{2} f_{A}, \\
f_{A}^{-1}=\left[(\omega-\Lambda)^{2}+\Gamma^{2}\right]^{2}, \\
F_{C D 1}=2 t^{2}\left[(\omega-\Lambda)^{2}-t^{2}-\Gamma^{2}\right] f_{C D}, \\
F_{C D 2}=2 t^{2} \Gamma(\omega-\Lambda) f_{C D}, \\
H_{A 1 \pm}=\left[(\omega-\Lambda)(\omega-\Lambda \pm t)-\Gamma^{2}\right] h_{A \pm}, \\
H_{A 2 \pm}=\Gamma(2 \omega-2 \Lambda \pm t) h_{A \pm}, \\
h_{A}^{-1}=\left[(\omega-\Lambda)^{2}+\Gamma^{2}\right]\left[(\omega-\Lambda \pm t)^{2}+\Gamma^{2}\right] .
\end{gathered}
$$

2. Функциям Грина $G_{1}(\omega) \approx g\left(1+3 g^{2} t^{2}\right), G_{-1}(\omega) \approx$ $\approx g\left(1+g^{2} t^{2}\right), G_{a}(\omega) \approx g_{a}\left(1+g g_{a} \tilde{t}^{2}\right), G_{2}(\omega) \approx g\left(1+2 g^{2} t^{2}\right.$ $\left.+g g_{a} \tilde{t}^{2}\right)$, полученным из выражений (6)-(9) в первом порядке по $t^{2} / \Gamma^{2}$, соответствуют следующие плотности состояний:

$$
\rho_{1}\left(\Omega_{g}\right) \approx \bar{\rho}_{g}\left(\Omega_{g}\right)\left(1+3 t^{2} \frac{3 \Omega_{g}^{2}-\Gamma^{2}}{\left(\Omega_{g}^{2}+\Gamma^{2}\right)}\right),
$$

$$
\begin{gathered}
\rho_{-1}\left(\Omega_{g}\right) \approx \bar{\rho}_{g}\left(\Omega_{g}\right)\left(1+t^{2} \frac{3 \Omega_{g}^{2}-\Gamma^{2}}{\left(\Omega_{g}^{2}+\Gamma^{2}\right)}\right), \\
\rho_{a}\left(\Omega_{a, g}\right) \approx \rho_{g}\left(\Omega_{a}\right)\left(1+\tilde{t}^{2} \frac{3 \Omega_{a} \Omega_{g}-\Gamma^{2}}{\left(\Omega_{a}^{2}+\Gamma^{2}\right)\left(\Omega_{g}^{2}+\Gamma^{2}\right)}\right), \\
\rho_{2}\left(\Omega_{a, g}\right) \approx \bar{\rho}_{g}\left(\Omega_{g}\right) \\
\times\left(1+2 t^{2} \frac{3 \Omega_{g}^{2}-\Gamma^{2}}{\left(\Omega_{g}^{2}+\Gamma^{2}\right)^{2}}+\tilde{t}^{2} \frac{\Omega_{g}^{2}+2 \Omega_{g} \Omega_{a}-\Gamma^{2}}{\left(\Omega_{g}^{2}+\Gamma^{2}\right)\left(\Omega_{a}^{2}+\Gamma^{2}\right)}\right),
\end{gathered}
$$

где $\quad \bar{\rho}_{a, g}(\omega)=\Gamma / \pi\left(\Omega_{a, g}^{2}+\Gamma^{2}\right), \quad \Omega_{g}=\omega-\Lambda, \quad \Omega_{a}=$ $=\omega-\varepsilon_{a}-\Lambda$.

\section{Список литературы}

[1] И.В. Антонова. ФТП 50, 67 (2016).

[2] Q. Sun, Y. Dai, Y. Ma, N. Yin, W. Wei, L. Yu, B. Huang. 2D Mater. 3, 035017 (2016).

[3] J. Zhang, W. Xie, X. Xu, S. Zhang, J. Zhao. Chem. Mater. 28, $5022(2016)$.

[4] G.C. Loh, R. Pandey. J. Mater. Chem. C 3, 5918 (2015).

[5] Z.-Y. Ong, G. Zhang, Y.-W. Zhang. Phys. Rev. B 93, 075406 (2016).

[6] С.Ю. Давыдов. ФТТ 60, 1815 (2018).

[7] С.Ю. Давыдов. Письма в ЖТФ 44, 21, 55 (2018).

[8] R. Taira, A. Yamanaka, S. Okada. Appl. Phys. Exp. 9, 115102 (2016).

[9] M.R. Mananghaya, G.N. Santos, D. Yu, C. Stampf. Sci. Rep. 7, 15727 (2017).

[10] Z.S. Sadeq, R.A. Muniz, J.E. Sipe. Phys. Rev. Mater. 2, 014001 (2018).

[11] С.Ю. Давыдов. ФТТ 61, 186 (2019).

[12] С.Ю. Давыдов. ФТП 53, 83 (2019).

[13] С.Ю. Давыдов. ФТТ 61, 610 (2019).

[14] С.Ю. Давыдов. Теория адсорбции: метод модельных гамильтонианов. Изд-во СПбГЭТУ „ЛЭТИ“ СПб., (2013). 235 c.; twirpx.com/file/1596114/

[15] С.Ю. Давыдов, А.А. Лебедев, О.В. Посредник. Элементарное введение в теорию наносистем. Изд-во „Лань“", СПб.: (2014).

[16] J. Cserti. Am. J. Phys. 68, 896 (2000).

[17] G. Jose, R. Malla, V. Srinivasan, A. Sharma, S. Gangadharaiah. arXiv: 1711.08204

[18] W.A. Harrison. Phys. Rev. B 31, 2121 (1983).

[19] T.O. Wehling, E. Şaşıoglu, C. Friedrich, A.I. Lichtenstein, M.I. Katsnelson, S. Blügel. Phys. Rev. Lett. 106, 236905 (2011).

[20] С.Ю. Давыдов. Письма в ЖТФ 39, 2,7 (2013).

[21] С.Ю. Давыдов. ЖТФ 84, 4, 155 (2014).

[22] С.Ю. Давыдов, С.В. Трошин. ФТТ 49, 1508 (2007).

[23] C. Berger, E. Conrad, W. De Heer. Epigraphene: epitaxial graphene on silicon carbide. G. Chiarotti, P. Chiaradia. Physics of Solid Surfaces, Subvol. B, Landolt-Börnstein: Numerical Data and Functional Relationships in Science and Technology — New Ser. Subvol. III/45B, 2017.

[24] С.Ю. Давыдов, А.В. Трошин. ФТТ 49, 723 (2007).

[25] С.Ю. Давыдов. ФТТ 58, 779 (2016).

[26] С.Ю. Давыдов. ФТТ 58, 1182 (2016).

Редактор К.В. Емщев 